\title{
Fistulectomy versus fistulotomy with marsupialisation in the treatment of low fistula-in- ano: a prospective randomized controlled trial
}

\author{
PHILLIPO L. CHALYA* and JOSEPH B. MABULA \\ Department of Surgery, Catholic University of Health and Allied Sciences-Bugando, Mwanza, Tanzania
}

\begin{abstract}
Low fistula-in-ano has traditionally been treated with fistulectomy and fistulotomy. Recent studies have shown that marsupialisation of the fistulotomy wounds can reduce the healing time. A randomized clinical trial was conducted at Bugando Medical Centre in north-western Tanzania between January 2008 and December 2012 to compare the efficacy of fistulectomy versus fistulotomy with marsupialisation in the treatment of low fistula in ano. Patients were randomized into two groups, namely Group A (fistulectomy) and Group B (fistulotomy with marsupialisation). A total of 162 patients were enrolled in the study and randomly assigned to Group A ( $n=82)$ and Group B $(n=80)$. Both groups were comparable with respect to age, sex ratio, duration of symptoms, type of fistula and radial distance of the external opening from the anal verge. The mean healing time was statistically significantly longer in group $A$ than in group $B(P=0.002)$. The duration of postoperative wound discharge was statistically significantly shorter in group $B$ than in group $A(P=0.012)$. There was no statistically significant difference in the mean operating time, mean operation wound size, mean postoperative pain score, rate of surgical site infection and postoperative hospital stay between the two groups $(P>0.05)$. None of the patients in either group had recurrence or was found to have anal incontinence. No differences in the extent of adverse effects of surgery on the physical, social and sexual lives of the patients in the two groups. We conclude that in comparison to a fistulectomy, a fistulotomy with marsupialisation results in faster wound healing and a shorter duration of wound discharge and should therefore be recommended as a standard surgical procedure in the treatment of low fistula-in-ano.
\end{abstract}

Keywords: Low fistula-in-ano, fistulectomy, fistulotomy, marsupialisation, Tanzania

\section{Introduction}

Fistula-in-ano is one of the most common benign anal conditions in daily surgical practice. It is defined as an epithelised abnormal tract connecting two surfaces, usually the rectal mucosa and perianal skin (Parks et al., 1976). Fistula-in-ano usually results from an anorectal abscess which bursts spontaneously or after inadequate surgery (Williams, 2004; Bhatti et al., 2011). Acute infection of the anal crypt leads to an anorectal abscess and fistula-in-ano represents the chronic form of this infection (Kodner et al., 1994; Bhatti et, al., 2011).

Different classifications have been put forward which categorize these fistulae into low or high, simple or complex, or according to their anatomy - inter-sphincteric, trans-sphincteric, and suprasphincteric or extra-sphincteric (Parks et al., 1976). Low fistula-in-ano open in to the anal canal below the anorectal ring and high fistula-in-ano open in to the anal canal at or above the anorectal ring (Bhatti et al., 2011). Two- thirds are posterior, one-third anterior (Bhatti et al., 2011). Studies have revealed that low type fistulae (low inter-sphincteric and low trans-sphincteric) are the commonest anal fistulae accounting for up to $90 \%$ of cases (Seow-Choen \& Nicholls, 1992; Bhatti et al., 2011; Jain et al., 2012).

The mainstay of treatment of fistula-in-ano is eradication of sepsis with preservation of anorectal function (Bhatti et al., 2011). Conventional surgical options for a low fistula-in-ano include a fistulotomy and a fistulectomy (Kronborg, 1985). A fistulectomy involves complete excision of the fistulous tract, thereby eliminating the risk of missing secondary tracts and providing complete tissue

\footnotetext{
* Correspondence: Phillipo L. Chalya; E-mail: drphillipoleo@yahoo.com
} 
for histopathological examination. A fistulotomy lays open the fistulous tract, thus leaving smaller unepithelised wounds, which hastens the wound healing. Recent studies have shown that marsupialisation of the fistulotomy wounds can reduce the healing time further (Ho et al., 1998; Jain et al., 2012). This randomized clinical trial was conducted at Bugando Medical Centre in Tanzania to compare the efficacy of fistulectomy versus fistulotomy with marsupialisation in the treatment of a low fistula-in-ano with an aim to evaluate fistulotomy with marsupialisation as an effective alternative to fistulectomy.

\section{Materials and Methods}

\section{Study area and design}

This was a prospective randomized clinical trial which was conducted in the general surgical wards of Bugando Medical Centre (BMC) and surgical outpatient clinic over a five-year period between January 2008 and December 2012. BMC is located in Mwanza City along the shore of Lake Victoria in north-western Tanzania. It is a tertiary care and teaching hospital for the Catholic University of Health and Allied Sciences-Bugando (CUHAS-Bugando) and has 1000 beds. BMC is one of the four largest referral hospitals in the country and serves as a referral centre for tertiary specialist care for a catchment population of approximately 13 million people from neighbouring regions.

\section{Study population}

All patients admitted in the surgical wards with a clinical diagnosis of a low fistula-in-ano were included in the study. Inclusion criteria were as follows: low fistula-in-ano; a single internal and a single external opening; the absence of a secondary tract. Patients with a recurrent fistula, patients with associated co-morbid conditions such as anal fissure, haemorrhoids, chronic colitis, and patients refusing consent for inclusion in study were excluded.

\section{Recruitment of patients}

Recruitment of patients to participate in the study was carried out in the general surgical wards and surgical outpatient clinic. Patients who met the inclusion criteria were enrolled into the study. All patients included in the study were interviewed to ascertain their clinical histories including presenting symptoms; duration of symptoms; and history of anorectal sepsis, previous surgery, and chronic illness. Inquiries were made to assess anal continence in each patient. All patients underwent detailed clinical examination to assess general health, presence of systemic disease, and anorectal pathology. The examination included perineal inspection, palpation, digital rectal examination, and proctoscopic evaluation. The distance of the external opening from the anal verge was measured using a plastic scale at the time of clinical examination.

\section{Randomization and blinding}

The patients included in the study were randomized into two groups according to whether fistulectomy or fistulotomy with marsupialisation was performed. A computer programme (random number generator, Microsoft Excel 5.0) was used to generate random number list, whereby patients were randomly divided into two groups ( $A$ and $B$ ). Group $A$ included patients who underwent a fistulectomy whereas Group B included group of patients that underwent a fistulotomy with marsupialisation. The randomization was performed by a computer consultant using computergenerated tables (www.randomization.com) having blocks of four patients in a 1:1 allocation ratio. No blinding was done in this study. 


\section{Interventions}

The patients were operated on under regional or general anaesthesia. Under anaesthesia, an anorectal examination was performed to verify the findings of the clinical examination. A dye study of the fistula tract was performed by placing moist gauze in the anal canal and injecting about $2 \mathrm{~mL}$ of methylene blue through the external opening. Staining of the gauze piece denoted patency of the fistula tract. A probe was gently passed into the fistulous tract through the external opening.

In the fistulectomy, a keyhole skin incision was made over the fistulous tract and encircled the external opening. The incision was deepened through the subcutaneous tissue, and the tract was removed from surrounding tissues. Towards the anal verge, fibres of the anal sphincters overlying the tract were divided (Farquahasan, 1971). While the tract was being removed, attention was paid to identifying secondary tracts, if any. Haemostasis was achieved.

In the fistulotomy with marsupialisation, the fistula tract was laid open over the probe placed in the tract. After the fistula tract had been laid open, the tract was curetted and examined for secondary extensions. Wound edges were sutured with the edge of fistula tract by using interrupted 3-0 chromic catgut sutures to marsupialize the operative wound from distal to proximal. The marsupialisation would prove difficult proximally where the ano-rectal mucosa had been friable. Haemostasis was achieved. The operating time for the procedure was calculated from the start of the dye test to the beginning of dressing of the postoperative wound. Patients in both groups were administered ciprofloxacin and metronidazole as perioperative antibiotics for a total duration of three days. Intramuscular Pethidine (50-100mg 8 hourly for 24 hours) followed by Diclofenac sodium (50 mg twice a day) for a total duration of 3 days were prescribed as analgesics. The patients were discharged on the first postoperative day. The patients were advised regarding oral medication, maintenance of local hygiene, sitz bath after defecation, dressings, and regular follow-ups.

The initial postoperative assessment was undertaken at twenty-four hours following surgery. The severity of postoperative pain was assessed on a scale of 0 to 10 with help of the visual analogue scale (VAS). Patients were asked about anal incontinence. Development of incontinence was assessed using the three-point Lickert scale (0, never; 1, sometimes; 2, always) according to inability to distinguish between gas and stool, difficulty in holding gas, and soiling of undergarments (García-Aguilar et al., 2000). All patients were followed up for a total duration of twelve weeks during the postoperative period. Patients were followed up at weekly intervals for the initial 6 weeks and at 2-week intervals for another 6 weeks. During each follow-up visit, the patient was assessed for postoperative pain, wound infection, and anal incontinence. Postoperative wound discharge was defined as a non-infected sero-sanguinous secretion from the open postoperative wound. Wound infection was defined as the presence of erythema, induration surrounding the wound or constitutional symptoms such as fever. Time required for complete healing of the postoperative wound, which was defined as the time for complete healing to take place with no area with an unepithelised surface, was noted. The patients were observed for recurrence of the fistula during the follow-up period. No patients were lost during the follow-up period.

Patients were interviewed to assess their levels of satisfaction with respect to the treatment. The patients were requested to report whether the treatment affected their lifestyles during the postoperative period in terms physical, social and sexual activities. For purposes of comparison, healing time was the primary outcome while size of the operative wound, operating time, postoperative pain, postoperative incontinence, patient satisfaction on the Lickert scale in terms of physical, social and sexual activities, and recurrence were secondary outcomes. 


\section{Data analysis}

Statistical data analysis was performed using the SPSS version 17.0 (SPSS Inc., Chicago, IL, USA). Qualitative data from the two groups were compared using the Chi-square test or Fischer's exact test while quantitative data were compared using the Mann-Whitney $\mathrm{U}$ test.

\section{Ethical consideration}

Ethical approval to conduct the study was obtained from the CUHAS/BMC Joint Institutional Ethic Review Committee before the commencement of the study. An informed written consent was sought from patients or relatives.

\section{Results}

During the period of study, a total of 172 patients with low fistula-in-ano were eligible for the study. Out of these, ten patients were excluded from the study due to failure to meet the inclusion criteria. Thus, 162 patients were enrolled in the study. There were 150 (92.6\%) male and 12 (7.4\%) female with a male to female ratio of 12.5: 1. The age of patients at presentation ranged from 19 to 76 years with a median age of 38.0 years. Eighty-two patients were randomized to Group A and 80 patients to group $B$ (Figure 1).

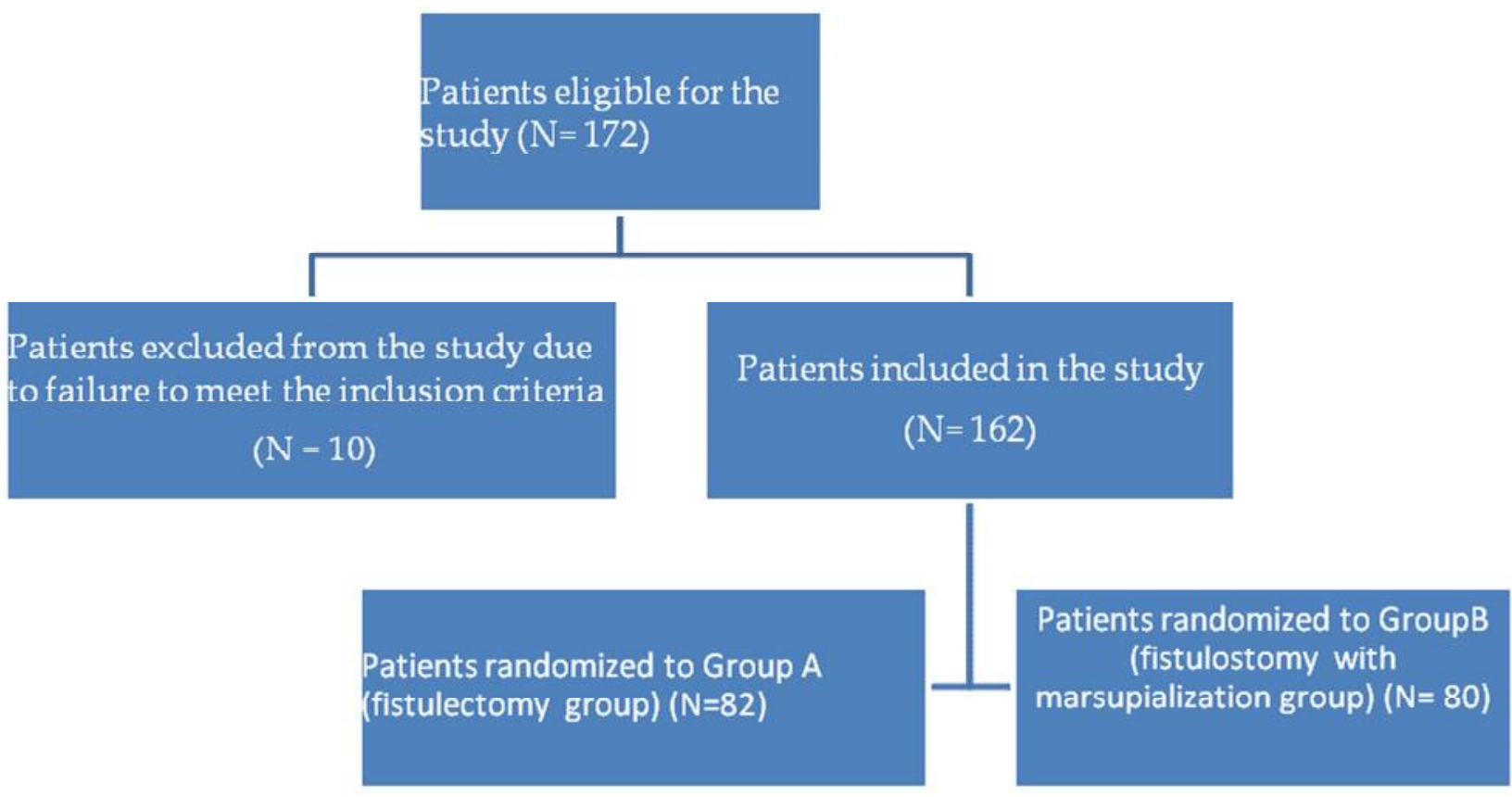

\section{Figure 1: Flow chart of patients}

Group A (fistulectomy group) consisted of 76 males and 6 females ( $M: F=12.7: 1$ ) whereas Group $B$ (fistulotomy with marsupialisation group) comprised of 74 males and 6 females $(M: F=12.3: 1)$. There was no statistically significant difference in the gender between the two groups $(P=0.854)$. The mean age in Group A was $37.8 \pm 16.4$, while it was $38.6 \pm 13.2$ in Group $B$. There was no significant difference between two groups with respect to age $(P=0.715)$. The commonest symptom was the purulent/watery discharge from the external opening of the fistula in $138(85.2 \%)$ patients. The pain was present in $65(40.1 \%)$ patients; and swelling near anus was present in $16(38.3 \%)$ patients. The mean durations of symptoms in groups A and B, respectively, were $7.4 \pm 3.6$ days and $8.2 \pm 2.2$ days 
$(P=0.231)$. The mean radial distances of the external opening from the anal verge were $2.5 \pm 1.1 \mathrm{~cm}$ and $1.9 \pm 0.6 \mathrm{~cm}$ in groups $A$ and $B$, respectively $(P=0.823)$. Both groups were comparable with respect to age, sex ratio, duration of symptoms, type of fistula and radial distance of the external opening from the anal verge (Table 1). Subcutaneous fistula-in-ano was the most common type in 134 (82.7\%) patients. Inter-sphincteric and trans-sphincteric fistula-in-ano were recorded in 16 (9.9\%) and $12(7.4 \%)$ patients, respectively. There was no significant statistical difference between the two groups with respect to the type of fistula $(P>0.05)$

Table 1: Preoperative and operative characteristics among the two groups

\begin{tabular}{|c|c|c|c|}
\hline Characteristics & Group A & Group B & P-value \\
\hline Mean age (in years) & $37.8 \pm 16.4$ & $38.6 \pm 13.2$ & 0.715 \\
\hline Sex ratio (male/female) & $12.7: 1$ & $12.3: 1$ & 0.854 \\
\hline Mean durations of symptoms (in months) & $7.4 \pm 3.6$ & $8.2 \pm 2.2$ & 0.231 \\
\hline $\begin{array}{l}\text { Mean radial distances of the external opening from the } \\
\text { anal verge (in } \mathrm{cm} \text { ) }\end{array}$ & $2.5 \pm 1.1$ & $1.9 \pm 0.6$ & 0.823 \\
\hline $\begin{array}{l}\text { Type of fistula (Subcutaneous/ Inter-sphincteric/ Trans- } \\
\text { sphincteric) }\end{array}$ & $66 / 9 / 7$ & $68 / 7 / 5$ & 0.632 \\
\hline
\end{tabular}

The mean operating time in Group A was $28.4 \pm 6.7$ minutes, whereas in Group A was $29.2 \pm 8.4$ minutes. The difference between the two groups with respect to the mean operating time was not statistically significant $(P=0.123)$. The mean operation wound size was $2.4 \pm 0.2 \mathrm{~cm}^{2}$ in group $A$, while it was $1.2 \pm 0.1 \mathrm{~cm}^{2}$ in group $B(P=0.542)$. Postoperative wounds ceased to ooze significantly earlier in group $B(2.6 \pm 1.2$ weeks $)$ than in group $A(4.3 \pm 1.4$ weeks $)(P=0.012)$. The mean postoperative VAS score at various follow-up times was higher in Group B than in Group A. There was no significant statistical difference between the two groups $(P>0.05)$ (Table 2$)$.

Table 2: The mean postoperative visual analogue scale (VAS) score among the two groups

\begin{tabular}{llllllll}
\hline Mean VAS score & $\mathbf{2 4}$ hours & $\mathbf{1}$ week & $\mathbf{2}$ week & $\mathbf{3}$ week & 4 week & $\mathbf{8}$ week & $\mathbf{1 2}$ week \\
\hline Group A & 4.2 & 2.0 & 1.3 & 0.9 & 0.1 & 0 & 0 \\
Group B & 4.8 & 2.7 & 2.4 & 1.8 & 0.4 & 0.1 & 0 \\
p-value & 0.089 & 0.335 & 0.342 & 0.453 & 0.564 & 0.807 & 1.00 \\
\hline
\end{tabular}

Surgical site infection was recorded in 56 patients giving an overall surgical site infection rate of 34.7\%. The surgical site infection rates in Group A and Group B were 32.6\% and 34.9\% respectively. The difference between the two groups in terms of the surgical site infection rates was not statistically significant $(P=0.293)$. The mean healing time was longer in Group $A$ than in group $B$ (36.4 \pm 12.8 versus $28.6 \pm 16.3$ days). This difference in healing time reached statistical significance with a p-value of 0.002 . None of the patient in either group had recurrence or was found to have incontinence. The mean length hospital stay (LOS) for the entire group was $4.8 \pm 1.3$. The mean LOS in the Group A and Group B were 3.9 \pm 0.9 and $4.2 \pm 1.6$ days respectively. The mean LOS did not differ significantly between the two groups $(p=0.672)$. No differences in the extents of adverse effects of surgery on the physical, social and sexual lives of the patients in the two groups (Table 3 ). 
Table 3: Adverse effects on the lifestyles of fistulectomy (Group A) and fistulotomy-withmarsupialisation (Group B) patients

\begin{tabular}{lll}
\hline Adverse effect on lifestyle & Group $\mathbf{A}(\mathbf{n}=\mathbf{8 2}$ & Group $\mathbf{B}(\mathbf{n}=\mathbf{8 0})$ \\
\hline Physical activity & & 69 \\
- Not at all & 70 & 11 \\
- To some extent & 12 & 0 \\
- Greatly & 0 & 70 \\
Social activity & & 8 \\
- Not at all & 74 & 2 \\
- To some extent & 7 & \\
- Greatly & 1 & 72 \\
Sexual activity & & 7 \\
- Not at all & 76 & 1 \\
- To some extent & 6 & \\
- Greatly & 0 &
\end{tabular}

\section{Discussion}

The fistula-in-ano has been a common surgical ailment reported since the time of Hippocrates. However, the management of the condition has been rarely documented in Tanzania (Malik \& Nelson, 2008). Various surgical treatments, including a fistulotomy, a fistulectomy, a seton and more complex sphincter-preserving procedures such as fibrin glue injection and fistula plug insertion, are currently been used depending on the type of fistula and the patient's continence (Pescatori et al., 2006; Malik \& Nelson, 2008).

Traditionally, fistulectomy and fistulotomy had commonly been used in the treatment of low fistula-in-ano (Kronborg, 1985). Recent studies have postulated that marsupialisation after fistulotomy leaves less raw unepithelialised tissue in the fistulotomy wound, thereby resulting in less postoperative blood loss and faster wound healing (Pescatori et al., 2006; Malik \& Nelson, 2008; Bhatti et al., 2011; Jain et al., 2012). However, this added procedure cannot prevent postoperative deformity and showed no improved functional outcome. Marsupialisation is not regarded as an essential procedure and many surgeons are reluctant to perform it even though it can facilitate faster wound healing (Malik \& Nelson, 2008). Therefore, whether to implement marsupialisation over a fistulotomy depends on the surgeon's preference. The patient satisfaction after surgical treatment for anal fistula depends on factors like period of hospitalization, postoperative pain and bleeding, return to routine activity, wound care, wound healing time, interference with the anal continence and the recurrence of the disease (Bhatti et al., 2011; Jain et al, 2012).

Several randomized clinical trials have compared the efficacy of fistulectomy versus fistulotomy with marsupialisation in the treatment of low fistula-in-ano (Pescatori et al., 2006; Bhatti et al., 2011; Jain et al., 2012). In agreement with other clinical trials (Pescatori et al., 2006; Jain et al, 2012), our study has demonstrated no significant difference in the operating times for the fistulectomy and fistulotomy with marsupialisation groups. This observation can be explained by the fact that the fistulectomy operation requires dissection of the fistula tract from the surrounding tissues, followed by coagulation of bleeding to control homeostasis. During a fistulotomy with marsupialisation, the fistula tract is laid open, so dissection of the fistula tract is not required, but several minutes are required to suture the edges of the laid-open fistula tract to the skin incision. Thus, both procedures are likely to require almost similar times. 
In this study, the mean operation wound size was smaller in fistulotomy with marsupialisation group than in fistulectomy group but the differences were not statistically significant. This finding agrees with other randomized clinical studies (Pescatori et al., 2006; Bhatti et al., 2011; Jain et al, 2012). This difference can be explained by the fact that removal of complete track and adjacent tissues in fistulectomy results in larger wound size as compared to excision of lesser amount of tissue in fistulotomy which results in smaller wound.

In keeping with other randomized clinical trials (Pescatori et al., 2006; Jain et al., 2012), the present study showed no significant difference between the two groups in the mean postoperative VAS score at various follow-up times. This observation is at variant with Bhatti et al. (2011) who reported more postoperative pain in fistulectomy group than in fistulotomy with marsupialisation group.

Our study demonstrated that fistulotomy with marsupialisation was associated with significantly lesser postoperative bleeding as compared to the fistulectomy and this difference reached statistical significance. The results of this study are also in agreement with that of Jain et al. (2012) which compared fistulectomy and fistulotomy with marsupialisation in the treatment of simple anal fistulae. Similar finding was also reported by Pescatori et al. (2006).

In the present study, the mean healing time was statistically significantly longer in group A than in group B which similar to findings from other randomized clinical trials (Pescatori et al., 2006; Jain et al., 2012). This finding can be explained by the fact that the mean operation wound size in this study was smaller in fistulotomy with marsupialisation group than in fistulectomy group, though the difference was not statistical significant. Further in the case of the fistulotomy with marsupialisation, the fistula tract, which could have been epithelised to varying extent, formed the floor of the wound. In addition, the present study showed no statistically significant differences in the rates of postoperative wound infection and postoperative hospital stay between the two groups which is in consistent with other trials (Lindsey et al, 2002; Jain et al, 2012).

In a randomized clinical trial by Kronborg (1985), the recurrence rates following fistulectomy and fistulotomy were reported to be $9.52 \%$ and $12.5 \%$, respectively, during a follow-up period of 12 months. In our series, no recurrence was reported in any patient in either group for a follow-up period of 12 weeks. However, the duration of observation in the present study was not sufficient to draw any definite correlation with respect to recurrence.

Most randomized clinical trials have demonstrated the development of anal incontinence after fistulectomy and fistulotomy with marsupialisation in the treatment of low fistula-in-ano (Kronborg, 1985; Ho et al., 1998; Lindsey et al., 2002). None of the patients in either group was found to have anal incontinence during a follow-up period. This observation is logical as all the internal openings were located in the lower anal canal in our patients. In agreement with other randomized clinical studies (Lindsey et al., 2002; Jain et al., 2012), our study demonstrated no differences in the extents of adverse effects of surgery on the physical, social and sexual lives of the patients in the two groups.

In conclusion, this study demonstrated shorter wound healing time and shorter duration of postoperative wound discharge following a fistulotomy with marsupialisation in comparison to a fistulectomy and should therefore be recommended as a standard surgical procedure in the treatment of low fistula-in-ano. However, due to small sample size and short period of follow up, the findings of the present study need to be substantiated further with studies involving larger sample sizes and longer period of follow-up. 


\section{Acknowledgements}

We are grateful to all those who provided care to our patients and those who provided support in the preparation of this manuscript. Special thanks go to our research assistants for their support in data collection.

\section{References}

Bhatti, Y., Fatima, S., Shaikh, G.S. \& Shaikh S. (2011) Fistulotomy versus fistulectomy in the treatment of low fistula in ano. Rawal Medical Journal 36, 284-286

Farquahasan, E.L. (1971) Operations in rectum and anal canal: Fistula-in-ano. In: Rintoul HF, editor. Textbook of Operative Surgery. 5th ed. Edinburgh: Churchill Livingstone; 732-733.

García-Aguilar, J., Davey, C.S., Le, C.T., Lowry, A.C. \& Rothenberger, D.A. (2000) Patient satisfaction after surgical treatment for fistula-in-ano. Diseases of Colon and Rectum 43, 1206-1212

Ho, Y.H., Tan, M., Leong, A.F. \& Seow-Choen, F. (1998) Marsupialization of fistulotomy wounds improves healing: a randomized controlled trial. British Journal of Surgery 85, 105-7

Jain, B.K., Vaibhaw, K., Garg, P.K., Gupta, S. \& Debajyoti Mohanty, D. (2012) Comparison of a Fistulectomy and a Fistulotomy with Marsupialization in the Management of a Simple Anal Fistula: A Randomized, Controlled Pilot Trial. Journal of the Korean Society of Coloproctology $28,78-82$.

Kodner, I.J., Fry, R.D., Fleshman, J.W. \& Birnbaum, E.H. (1994) Colon rectum and anus. In: Schwartz SI, Shires GT, Spencer FC, editors. Principles of Surgery. 6th ed. New York: Mc Gaw-Hill; 1921306.

Kronborg, O. (1985) To lay open or excise a fistula-in-ano: a randomized trial. British Journal of Surgery 72,970

Lindsey, I., Smilgin-Humphreys, M.M., Cunningham, C., Mortensen, N.J, George, B.D. (2002) A randomized, controlled trial of fibrin glue vs. conventional treatment for anal fistula. Diseases of the Colon and Rectum 45, 1608-1615.

Malik, A.I. \& Nelson, R.L. (2008) Surgical management of anal fistulae: a systematic review. Colorectal Diseases 10, 420-30

Parks, A.G., Gordon, P.H. \& Hardcastle, J.D. (1976) A classification of fistula-in-ano. British Journal of Surgery 63, 1-12.

Seow-Choen, F. \& Nicholls, R.J. (1992) Anal fistula. British Journal of Surgery 79, 197- 205

Pescatori, M., Ayabaca, S.M., Cafaro, D., lannello, A. \& Magrini, S. (2006) Marsupialization of fistulotomy and fistulectomy wounds improve healing and decreases bleeding: a randomized controlled trial. Colorectal Diseases 8, 11-14.

Williams, N.S. (2004) The anus and anal canal. In: Russell, R.C.G., Williams, N.S., Bulstrode, C.J.K., editors. Bailey \& Loves Short Practice of Surgery. 24th ed. London: Edward Arnold 1242-71. 